\title{
High resolution mid-infrared imaging of $\mathrm{W} 3(\mathrm{OH})$
}

\author{
B. Stecklum ${ }^{1}$, B. Brandl ${ }^{2}$, Th. Henning ${ }^{3}$, I. Pascucci ${ }^{3}$, T. L. Hayward ${ }^{4}$, and J. C. Wilson ${ }^{2}$ \\ 1 Thüringer Landessternwarte Tautenburg, Sternwarte 5, 07778 Tautenburg, Germany \\ 2 Center for Radiophysics \& Space Research, Cornell University, Ithaca, NY 14853, USA \\ ${ }^{3}$ Max-Planck-Institut für Astronomie, Königstuhl 17, 69117 Heidelberg, Germany \\ ${ }^{4}$ Gemini Observatory, 670 N. A'ohoku Place, Hilo, HI 96720, USA
}

Received 29 April 2002 / Accepted 3 July 2002

\begin{abstract}
We present results of our diffraction-limited mid-infrared imaging of the massive star-forming region W3(OH) with SpectroCam-10 on the 5-m Hale telescope at wavelengths of 8.8, 11.7, and $17.9 \mu \mathrm{m}$. The thermal emission from heated dust grains associated with the ultracompact HII region $\mathrm{W} 3(\mathrm{OH})$ is resolved and has a spatial extent of $\sim 2^{\prime \prime}$ in the $N$ band. We did not detect the hot core source $\mathrm{W} 3\left(\mathrm{H}_{2} \mathrm{O}\right)$ which implies the presence of at least $12 \mathrm{mag}$ of extinction at $11.7 \mu \mathrm{m}$ towards this source. These results together with other data were used to constrain the properties of $\mathrm{W} 3(\mathrm{OH})$ and $\mathrm{W} 3\left(\mathrm{H}_{2} \mathrm{O}\right)$ and their envelopes by modelling the thermal dust emission.
\end{abstract}

Key words. stars: formation, circumstellar matter - ISM: dust, extinction - infrared: stars

\section{Introduction}

The "hot cores" revealed in recent years by molecular line investigations are small $(\lessgtr 0.1 \mathrm{pc})$, very dense $\left(n \gtrsim 10^{7} \mathrm{~cm}^{-3}\right)$, and hot $(>100 \mathrm{~K})$ entities of giant molecular clouds (e.g., Cesaroni et al. 1998). They are considered to be the likely birthplaces of massive stars (e.g., Garay \& Lizano 1999; Kurtz et al. 2000). Hot cores are frequently associated with ultracompact HII regions (UCHIIs) which are more evolved and accessible to near-infrared (NIR) studies of their stellar population (e.g., Feldt et al. 1998; Henning et al. 2001). Although the temperatures and sizes of hot cores suggest that they might be conspicuous objects in the infrared sky, extinction in the mid-infrared (MIR, $N(10 \mu \mathrm{m})$ and $Q(20 \mu \mathrm{m})$ bands) caused by the large column densities $\left(N\left(\mathrm{H}_{2}\right) \gtrsim 10^{23} \mathrm{~cm}^{-2}\right)$ must not be neglected.

Conclusions concerning the heating and the stellar content of hot cores have to be based on the knowledge of their luminosity. This quantity is difficult to estimate since the immediate neighbourhood of UCHIIs often leads to source confusion, especially in the far-infrared (FIR) range where these objects emit most of their energy and the angular resolution of spaceborn observations is as yet poor. Radio interferometry at $\mathrm{mm} / \mathrm{submm}$ wavelengths allows to separate the dust continuum emission of the hot core from the free-free radiation of the adjacent UCHII. Such measurements were used to constrain models of hot cores (Osorio et al. 1999). High-resolution ground-based MIR observations, on the other hand, provide information on the spectral energy distribution (SED) shortward of the peak flux. A corresponding study of the IRc2 source in

Send offprint requests to: B. Stecklum, e-mail: stecklum@tls-tautenburg.de the Orion BN/KL complex (Gezari et al. 1998) illustrates their importance. More recently, De Buizer et al. (2002) were able to detect MIR emission from the hot core G29.96-0.02 with a morphology similar to that of the warm ammonia. We performed high resolution MIR imaging of hot cores, including $\mathrm{W} 3\left(\mathrm{H}_{2} \mathrm{O}\right)$ and the neighbouring $\mathrm{UCH}$ II $\mathrm{W} 3(\mathrm{OH})$, in order to measure their flux densities or to provide at least upper limits. While results for G10.47+0.03 will be the subject of a forthcoming paper (Pascucci et al., in prep.), we present here our findings for $\mathrm{W} 3\left(\mathrm{H}_{2} \mathrm{O}\right)$ and the $\mathrm{UCH}$ II $\mathrm{W} 3(\mathrm{OH})$.

The UCHII $\mathrm{W} 3(\mathrm{OH})$ is very well-studied in the radio domain by continuum and molecular line observations. It is located at the distance of $2.2 \mathrm{kpc}$ (Humphreys 1978) and harbours numerous $\mathrm{OH}$ masers. The hot core $\mathrm{W} 3\left(\mathrm{H}_{2} \mathrm{O}\right)$, also known as Turner-Welch object (TW, Turner \& Welch 1984), is situated $\sim 6$ " east of $\mathrm{W} 3(\mathrm{OH})$. This enigmatic source shows an outflow traced by the proper motion of $\mathrm{H}_{2} \mathrm{O}$ masers (Alcolea et al. 1992) and is associated with a double-sided radio continuum jet, presumably of synchrotron nature (Reid et al. 1995). Recent interferometric imaging at $220 \mathrm{GHz}$ by Wyrowski et al. (1999) revealed another source in the immediate neighbourhood of $\mathrm{W} 3\left(\mathrm{H}_{2} \mathrm{O}\right)$, suggesting that the region harbours a cluster of protostars. The thermal infrared emission from $\mathrm{W} 3(\mathrm{OH})$ has been studied by Keto et al. (1992) using one of the first MIR array cameras (Arens et al. 1987). Keto et al. claimed the detection of $\mathrm{W} 3\left(\mathrm{H}_{2} \mathrm{O}\right)$ at the wavelength of $12.2 \mu \mathrm{m}$ with a flux density of $45 \pm 10 \mathrm{mJy} . \mathrm{W} 3\left(\mathrm{H}_{2} \mathrm{O}\right)$ and $\mathrm{W} 3(\mathrm{OH})$ were the subject of a continuum and molecular line study of van der Tak et al. (2000). Since their results are based on single-dish data which do not resolve the two objects, they cannot be directly compared to our model presented below. 


\section{Observations}

The observations were performed using SpectroCam-10, the Cornell-built 8-13 $\mu \mathrm{m}$ spectrograph/camera (Hayward et al. 1993 ) on the 5-m Hale telescope ${ }^{1}$. SpectroCam's detector is a Rockwell $128 \times 128 \mathrm{Si}$ :As BIB array. In camera mode, the pixel scale is 0.25 with a circular field of view of $16^{\prime \prime}$. We applied filters with central wavelengths and bandwidths (in parentheses) of $8.8(1.0), 11.7(1.0)$, and $17.9(0.5) \mu \mathrm{m}$. The imaging observations were performed on 1998 December 27th using the common chopping/nodding technique with a chopper throw of $20^{\prime \prime}$ in north-south direction. The wavelet filtering algorithm of Pantin \& Starck (1996) was applied to the images. This algorithm is useful for recovering faint extended emission otherwise hidden in the noise. The high dynamic range of the individual frames allowed the application of a shift-and-add procedure which includes resampling, yielding a final pixel scale of 0 . $^{\prime} 125$. To enlarge our field of view, we observed two positions at $11.7 \mu \mathrm{m}$, the first centered on $\mathrm{W} 3(\mathrm{OH})$ and the second offset to the east by $10^{\prime \prime}$. Lastly, the frames were mosaicked.

The astrometry is based on the radio position of $\mathrm{W} 3(\mathrm{OH})$ which is assumed to coincide with the peak of the infrared emission.

The flux calibration was performed using $\alpha$ Tau as reference which was measured at about the same airmass (1.2) as the target. For this purpose, the photometric zero points from Cohen et al. (1992) and the photometry from Cohen et al. (1995) for $\alpha$ Tau were used. The observations were performed during photometric conditions, with an internal photometric error of less than $2 \%$ as estimated from the flux variation of the standard star. The $3 \sigma$ sensitivities ( $\mathrm{mJy} / \mathrm{beam}$ ) for the detection of point sources in the final images are as follows: $4(8.8 \mu \mathrm{m})$, $6(11.7 \mu \mathrm{m})$, and $93(17.9 \mu \mathrm{m})$.

\section{Results}

The prime results of our observations are contained in Fig. 1 which shows the $11.7 \mu \mathrm{m}$ image together with the $8.4 \mathrm{GHz}$ radio continuum contours from Wilner at al. (1999, beam size $0.2)$. The two infrared sources in Fig. 1 correspond to W3(OH) and the cometary UCHII situated north-east of it. A comparison with $\alpha$ Tau (Fig. 1 insert) shows that $\mathrm{W} 3(\mathrm{OH})$ is spatially well resolved in our diffraction-limited $11.7 \mu \mathrm{m}$ image (beam size $\left.0 .{ }^{\prime} 6\right)$. The lowest contour line of the infrared emission represents the $3 \sigma$ detection limit. Clearly, the flux from $\mathrm{W} 3\left(\mathrm{H}_{2} \mathrm{O}\right)$ is below our sensitivity limit. This result is in contradiction to the infrared detection of $\mathrm{W} 3\left(\mathrm{H}_{2} \mathrm{O}\right)$ claimed by Keto et al. (1992). We note that their conclusion is doubtful since the emission they associate with $\mathrm{W} 3\left(\mathrm{H}_{2} \mathrm{O}\right)$ peaks only $4^{\prime \prime}$ instead of $6^{\prime \prime}$ east of $\mathrm{W} 3(\mathrm{OH})$ (see their Fig. 1). Furthermore, their total flux of $45 \pm 10 \mathrm{mJy}$ contradicts with the fact that the emission well exceeds their lowest contour level of $100 \mathrm{mJy} / \square^{\prime \prime}$. Thus, we conclude that they did not detect $\mathrm{W} 3\left(\mathrm{H}_{2} \mathrm{O}\right)$ as well, and were confused by the north-eastern cometary UCHII.

\footnotetext{
${ }^{1}$ Observations at the Palomar Observatory were made as part of a continuing collaborative agreement between the California Institute of Technology and Cornell University.
}

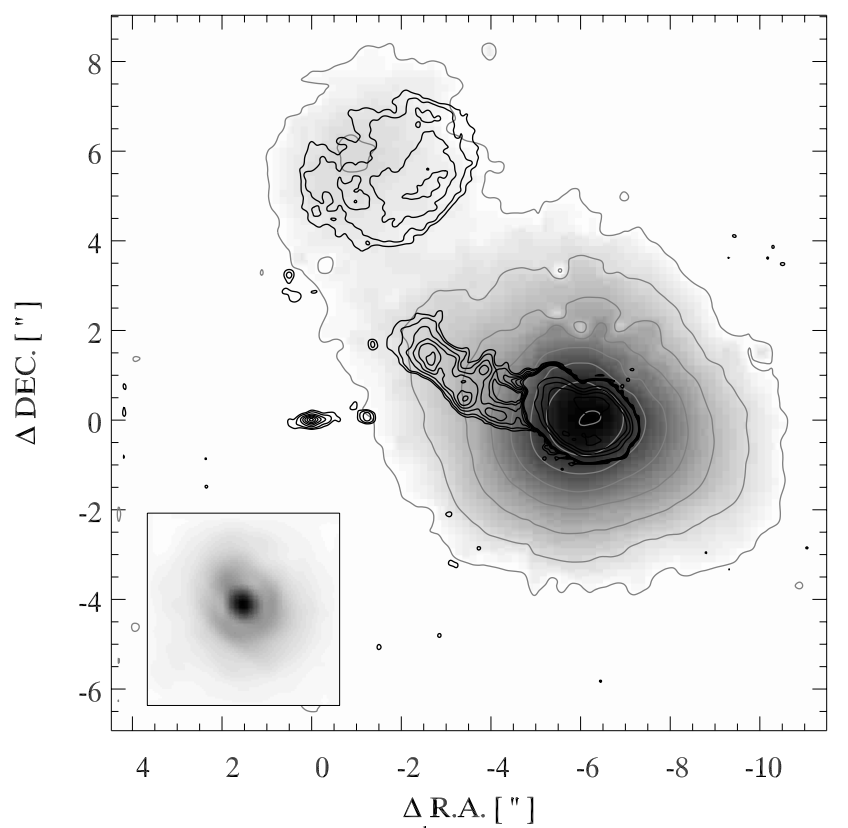

REFERENCE POS. R.A. $02^{\mathrm{h}} 27^{\mathrm{m}} 04^{\mathrm{s}} .71$ DEC. $+61^{\mathrm{o}} 52^{2} 24.6$ (2000)

Fig. 1. $11.7 \mu \mathrm{m}$ image of $\mathrm{W} 3(\mathrm{OH})$ with contours of the $8.4 \mathrm{GHz}$ radio continuum emission (black lines) from Wilner at al. (1999). The grey contours delineate the $11.7 \mu \mathrm{m}$ emission and are spaced by a factor of 2.5 starting at $3 \sigma\left(10 \mathrm{mJy} / \square^{\prime \prime}\right) . \mathrm{W} 3\left(\mathrm{H}_{2} \mathrm{O}\right)$ is the elongated radio source at the reference position. The lower-left insert shows the $11.7 \mu \mathrm{m}$ image of the reference star $\alpha$ Tau using a logarithmic brightness scale.

From Fig. 1 it is obvious that there is a good overall correspondence between the MIR and the $8.4 \mathrm{GHz}$ radio continuum emission. However, there are certain features which are different in both maps. The north-eastern trail from $\mathrm{W} 3(\mathrm{OH})$ is more confined in the radio continuum and only marginally indicated by the infrared contours. The peak of the radio emission from the north-eastern UCHII is closer towards $\mathrm{W} 3(\mathrm{OH})$ than its infrared maximum. This is presumably caused by the extinction of a dust lane stretching from $\mathrm{W} 3\left(\mathrm{H}_{2} \mathrm{O}\right)$ to the north-west which can be seen in the $\mathrm{C}^{17} \mathrm{O}(1-0)$ map of Wyrowski et al. (1997).

For the northeastern UCHII the following fluxes (within a $3^{\prime \prime}$ aperture) were derived at the three wavelengths: $0.3,0.6$, and $6.1 \mathrm{Jy}$, respectively.

\section{Discussion}

\subsection{The UCHII $\mathrm{W} 3(\mathrm{OH})$}

The $F W H M$ of the MIR emission from $\mathrm{W} 3(\mathrm{OH})$ was derived from Gaussian fits, taking the size of the diffraction-limited beam into account. The sizes and flux densities of $\mathrm{W} 3(\mathrm{OH})$ are listed in Table 1. The fluxes are based on an aperture of 4 " diameter. The extent of the MIR emission originating from the thermal emission of heated dust grains exceeds that of the $8.4 \mathrm{GHz}$ radio continuum ( $\left.F W H M 1^{\prime \prime} .52\right)$, indicating that the warm dust is more extended than the ionized gas. The variation of the angular size in dependence on wavelength can be approximated as $F W H M(\lambda) \sim \lambda^{0.6 \pm 0.2}$ and results from the decline 
Table 1. SpectroCam-10, IRAS-LRS, and MSX flux densities of $\mathrm{W} 3(\mathrm{OH})$.

\begin{tabular}{cccc}
\hline $\begin{array}{c}\text { Wavelength } \\
{[\mu \mathrm{m}]}\end{array}$ & $\begin{array}{c}\text { Peak flux } \\
{[\mathrm{Jy}] / \square^{\prime \prime}}\end{array}$ & $\begin{array}{c}\text { Total flux } \\
{[\mathrm{Jy}]}\end{array}$ & $\begin{array}{c}F W H M \\
{\left[{ }^{\prime \prime}\right]}\end{array}$ \\
\hline 8.8 & 2.6 & 6.5 & 1.96 \\
11.7 & 7.7 & 22.4 & 2.08 \\
17.9 & 19.5 & 93.0 & 2.88 \\
\hline IRAS-LRS & Total flux & MSX & Total flux \\
{$[\mu \mathrm{m}]$} & {$[\mathrm{Jy}]$} & {$[\mu \mathrm{m}]$} & {$[\mathrm{Jy}]$} \\
\hline 8.8 & 24.9 & 8.28 & 11.1 \\
11.7 & 24.1 & 12.13 & 36.7 \\
17.9 & 89.1 & 14.65 & 77.1 \\
& & 21.41 & 332.6 \\
\hline
\end{tabular}

of the temperature with increasing distance from the heating $\operatorname{star}(\mathrm{s})$.

The comparison of our flux densities of $\mathrm{W} 3(\mathrm{OH})$ with other estimates allows conclusions on the influence of different beam sizes. For this purpose, we retrieved the IRAS-LRS spectrum, identified $\mathrm{W} 3(\mathrm{OH})$ in the MSX point source catalog (Egan et al. 1999), and retrieved an ISO-LWS spectrum from the data archive.

The LRS spectrum was integrated according to the applied passbands. It is obvious that the $8.8 \mu \mathrm{m}$ fluxes given in Table 1 considerably exceed our value. This can be explained by ubiquitous emission attributed to Polycyclic Aromatic Hydrocarbons (PAHs) surrounding the UCHII which strongly contributes to the flux in the large apertures of IRAS and MSX. Pronounced 7.7 and $8.6 \mu \mathrm{m}$ PAH bands can be misleading in ground-based derivations of the optical depth of the $9.7 \mu \mathrm{m}$ silicate feature (e.g., Roelfsema et al. 1996).

\subsection{The hot core $W 3\left(\mathrm{H}_{2} \mathrm{O}\right)$}

Our attempt to detect $\mathrm{W} 3\left(\mathrm{H}_{2} \mathrm{O}\right)$ in the IR was stimulated by the presence of an outflow. Generally, IR emission can escape in outflow lobes primarily due to scattering (e.g., Fischer et al. 1996). An example is NGC6334 I(N), a presumed high-mass Class 0 object, for which Sandell (Sandell 2001) rendered the detection of IR emission impossible because of the extremely high extinction derived from $\mathrm{mm} / \mathrm{submm}$ maps. However, this source is associated with NIR emission (Tapia et al. 1996; Megeath \& Tieftrunk 1999) obviously originating from the blue-shifted lobe of its outflow.

The flux densities from $\mathrm{W} 3\left(\mathrm{H}_{2} \mathrm{O}\right)$ in the absence of any intervening absorbing matter can be estimated from the temperature map given by Wyrowski et al. (1997). The expected peak surface brightness amounts to $2170 \mathrm{Jy} / \square^{\prime \prime}$ at $11.7 \mu \mathrm{m}$. Together with our $3 \sigma$ sensitivity, this yields a lower limit to the extinction at this wavelength of 12 mag. Our failure to detect this source is consistent with the high molecular hydrogen column densities of $1 \ldots 3.5 \times 10^{24} \mathrm{~cm}^{-2}$ inferred from molecular line and continuum investigations (Turner \& Welch 1984;
Wyrowski et al. 1997). It suggests that the molecular outflow of $\mathrm{W} 3\left(\mathrm{H}_{2} \mathrm{O}\right)$ is very young, i.e. did not fully penetrate the hot core yet, and, in addition, might be in the plane of the sky. In fact, Fig. 1 from Wyrowski et al. (1999) shows that the jet is confined to the region of the hot core. The moderate expansion velocity of the $\mathrm{H}_{2} \mathrm{O}$ masers of $20 \mathrm{~km} \mathrm{~s}^{-1}$ (Alcolea et al. 1992) implies a dynamical timescale of only $500 \mathrm{yr}$ which is consistent with the upper limit on proper motions of the radio jet of $150 \mathrm{~km} \mathrm{~s}^{-1}$ (Wilner et al. 1999). These velocities are low compared to those of thermal radio jets (Anglada 1996) and indicate that the outflow is presumably slowed-down by the high-density environment.

\section{Modelling the dust emission}

One of the questions concerning $\mathrm{W} 3(\mathrm{OH})$ and $\mathrm{W} 3\left(\mathrm{H}_{2} \mathrm{O}\right)$ is related to their individual luminosities which directly translates to the nature of the internal heating sources. Although it is reasonable to assume that the internal heating is due to stars of intermediate or high mass, no direct confirmation of their presence exists. The luminosity might also, at least partly, result from accretion. The high column densities toward $\mathrm{W} 3\left(\mathrm{H}_{2} \mathrm{O}\right)$ render it very difficult to figure out whether the mass accretion is due to infall from a circumstellar disk or stellar mergers like in the scenario of Bonnell et al. (1998). The high optical depths will lead to a thermalization of the released energy irrespective of the accretion mechanism.

The derivation of the individual luminosities has to be based on the decomposition of the SED. While our measurements provide constraints for the MIR, the lack of spatial resolution in the FIR does not permit to separate both components. An upper limit of $\sim 1800 \mathrm{Jy}$ on the $50 \mu \mathrm{m}$ flux of $\mathrm{W} 3\left(\mathrm{H}_{2} \mathrm{O}\right)$ has been established by Campbell et al. (1989) from KAO scans. In the $\mathrm{mm} / \mathrm{submm}$ range, aperture synthesis measurements show that the $1.3 \mathrm{~mm}$ emission from $\mathrm{W} 3\left(\mathrm{H}_{2} \mathrm{O}\right)$ is due to dust radiation while free-free emission dominates the flux of $\mathrm{W} 3(\mathrm{OH})$ at this wavelength (Wyrowski et al. 1999). The ISO-LWS spectrum between 42 and $140 \mu \mathrm{m}$ can well be fitted by a modified black-body (35 $\mathrm{K}$ and $\kappa(v) \sim v^{2}$ ). The association of W3 $\left(\mathrm{H}_{2} \mathrm{O}\right)$ with cold dust suggests that the MIR/FIR excess in the ISO spectrum for $\lambda>140 \mu \mathrm{m}$ is presumably due to the hot core.

We modelled the thermal dust radiation from $\mathrm{W} 3(\mathrm{OH})$ and $\mathrm{W} 3\left(\mathrm{H}_{2} \mathrm{O}\right)$ in order to figure out how much the hot core contributes to the FIR excess seen in the ISO-LWS spectrum and which sensitivities are required for its detection in the MIR. The input SED is based on fluxes coming from this work, from the MSX point source catalogue at $21.4 \mu \mathrm{m}$ (see Table 1), from the IRAS-LSR and ISO-LWS spectra, and from Wyrowski et al. (1997) as well as Wilner et al. (1995) for the mm wavelengths. To solve the radiative transfer problem we used the 1D-code of Manske \& Henning (1998) assuming spherically symmetric shells like in previous works. As for the dust composition, we use graphite, silicate, and iron with optical constants taken from Dorschner et al. (1995) and Draine \& Lee (1984) together with a MRN-type size distribution (Mathis et al. 1977). The model parameters and the related references are given in Table 2: here $L_{*}$ is the stellar luminosity, $R_{\text {in }}$ and 
Table 2. Radiative transfer model parameters.

\begin{tabular}{llcll}
\hline \hline Source & $\begin{array}{l}L_{*} \\
{\left[10^{4} L_{\odot}\right]}\end{array}$ & $\begin{array}{c}R_{\text {in }} \\
{[\mathrm{AU}]}\end{array}$ & $\begin{array}{l}R_{\text {out }} \\
{[\mathrm{AU}]}\end{array}$ & $\begin{array}{l}M_{\mathrm{d}} \\
{\left[M_{\odot}\right]}\end{array}$ \\
\hline $\mathrm{W} 3(\mathrm{OH})$ & $8^{1}$ & $2270^{2,3}$ & $57000^{4}$ & 5.0 \\
$\mathrm{~W} 3\left(\mathrm{H}_{2} \mathrm{O}\right)$ & $2.4^{5,6}$ & 190 & $24000^{5}$ & $3.0^{7}$ \\
\hline
\end{tabular}

Ref: ${ }^{1}$ Wink et al. (1994), ${ }^{2}$ Chini et al. (1986), ${ }^{3}$ Campbell et al. (1989), ${ }^{4}$ Zeng et al. (1984), ${ }^{5}$ Osorio et al. (1999), ${ }^{6}$ Thompson (1984), ${ }^{7}$ Wyrowski et al. (1997).

$R_{\text {out }}$ are the inner and the outer radius of the envelope, and $M_{\mathrm{d}}$ is the dust mass. We underline that the inner radius of the dust shell of $\mathrm{W} 3(\mathrm{OH})$ coincides with the outer radius of the UCHII while for the hot core, $R_{\text {in }}$ is derived from the dust sublimation temperature $(\sim 1000 \mathrm{~K})$. To model the $\mathrm{W} 3\left(\mathrm{H}_{2} \mathrm{O}\right) \mathrm{SED}$ we follow Osorio et al. (1999) in adding an accretion luminosity of $4.2 \times 10^{4} L_{\odot}$ to the stellar luminosity and a free-fall density shell. For $\mathrm{W} 3(\mathrm{OH})$ the density profile that better fits our observations is Gaussian and yields an almost constant density distribution till $\sim 20000 \mathrm{AU}$. The resulting opacities at the $\mathrm{mm}$ wavelengths vary as $v^{1.3}$.

Figure 2 shows the individual SEDs as well as their superposition. Our model produces the following $F W H M$ s at the observed wavelengths: 1.'6 at $8.8 \mu \mathrm{m}, 2$.'0 at $11.7 \mu \mathrm{m}$ and $33^{\prime \prime} 1$ at $17.9 \mu \mathrm{m}$. They are quite in agreement with those given in Table 1 which supports the view that the parameters of the radiative transfer model are representative for the actual conditions. From the SED of $\mathrm{W} 3\left(\mathrm{H}_{2} \mathrm{O}\right)$ we conclude that deep observations in the $\mathrm{Q}$ band might be able to detect the MIR emission from the hot core.

\section{Summary}

1. The thermal infrared counterpart to the hot core source $\mathrm{W} 3\left(\mathrm{H}_{2} \mathrm{O}\right)$ was not detected at any wavelength of our observations. This revises the finding of Keto et al. (1992) which most probably resulted from confusing $\mathrm{W} 3\left(\mathrm{H}_{2} \mathrm{O}\right)$ with the UCHII northeast of $\mathrm{W} 3(\mathrm{OH})$. We derived a lower limit for the extinction at $11.7 \mu \mathrm{m}$ towards $\mathrm{W} 3\left(\mathrm{H}_{2} \mathrm{O}\right)$ of $12 \mathrm{mag}$.

2. Our diffraction-limited imaging resolved the thermal emission from $\mathrm{W} 3(\mathrm{OH})$ and clearly indicates a wavelength dependence of its apparent size.

3. The comparison of our $8.8 \mu \mathrm{m}$ flux to those measured with IRAS and MSX led to the conclusions that PAHs surround $\mathrm{W} 3(\mathrm{OH})$.

4. In accordance with our thermal infrared imaging and $\mathrm{mm} / \mathrm{submm}$ studies, the ISO-LWS spectrum of W3(OH) might be decomposed in two components with $\mathrm{W} 3(\mathrm{OH})$ being the hotter, more evolved, object while $\mathrm{W} 3\left(\mathrm{H}_{2} \mathrm{O}\right)$ dominating at mid/far-infrared wavelengths.

Acknowledgements. We thank S. Rinehart for assistance during the observations and D. Wilner for providing the $8.4 \mathrm{GHz}$ VLA map. This work was supported by the Deutsche Forschungsgemeinschaft grant Ste 605/18-1.

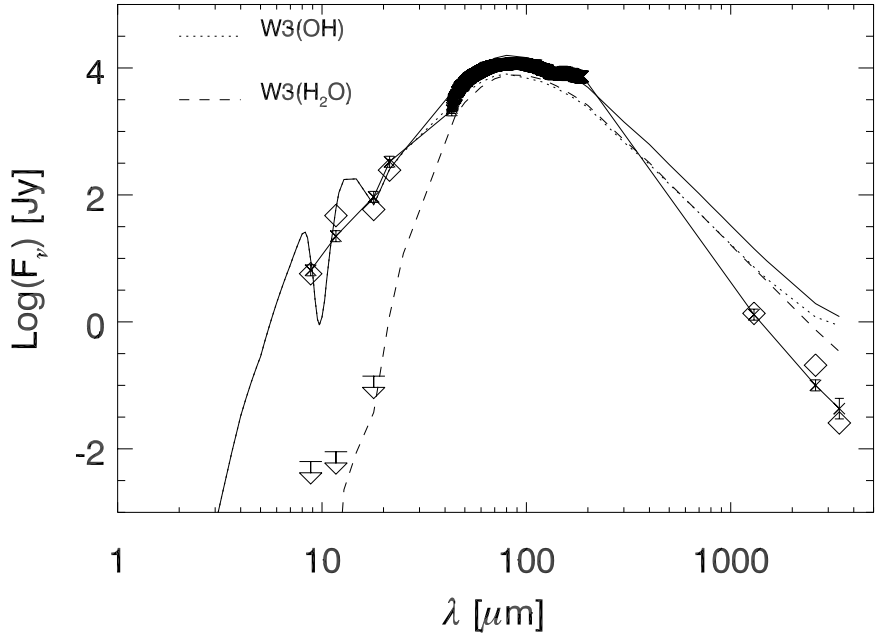

Fig. 2. SEDs of the individual sources $(\mathrm{W} 3(\mathrm{OH})$ - dotted line, W3 $\left(\mathrm{H}_{2} \mathrm{O}\right)$ - dashed line) and of their sum (solid line). Asterisks with error bars indicate the measured flux densities. Diamonds represent the model fluxes for the observational beam sizes. Arrows mark the detection limits for $\mathrm{W} 3\left(\mathrm{H}_{2} \mathrm{O}\right)$.

\section{References}

Alcolea, J., Menten, K. M., Moran, J. M., \& Reid, M. J. 1992, in Astrophysical Masers, ed. A. W. Clegg, \& Nedoluha (Berlin: Springer), 225

Anglada, G. 1996, in Radio Emission from the Stars and the Sun, ed. R. A. Taylor, \& J. M. Paredes (Astronomical Society of the Pacific Conf. Ser.), 93, 3

Arens, J. F., Jernigan, J. G., Peck, M. C., Dobson, C. A., \& Kilk, E. 1987, Appl. Opt., 26, 3846

Bonnell, I. A., Bate, M. R., \& Zinnecker, H. 1998, MNRAS, 298, 93

Campbell, M. F., Lester, D. F., Harvey, P. M., \& Joy, M. 1989, ApJ, 345,298

Cesaroni, R., Hofner, P., Walmsley, C. M., \& Churchwell, E. 1998, A\&A, 331, 709

Chini, R., Krügel, E., \& Kreysa, E. 1986, A\&A, 167, 315

Cohen, M., Walker, R. G., Barlow, M. J., \& Deacon, J. R. 1992, AJ, 104,1650

Cohen, M., Witteborn, F. C., Walker, R. G., Bregman, J. D., \& Wooden, D. H. 1995, AJ, 110, 275

De Buizer, J. M., Watson, A. M., Radomski, J. T., Piña, R. K., \& Telesco, C. M. 2002, ApJ, 564, L101

Dorschner, J., Begemann, B., Henning, Th., Jäger, C., \& Mutschke, H. 1995, A\&A, 300, 503

Draine, B. T., \& Lee, H. M. 1984, ApJ, 285, 89

Egan, M. P., Price, S. D., Moshir, M. M., et al. 1999, Air Force Laboratory Technical Report No. AFRL-VS-TR-1999-1522

Feldt, M., Stecklum, B., Henning, Th., et al. 1998, A\&A, 339, 759

Fischer, O., Henning, Th., \& Yorke, H. 1996, A\&A, 308, 863

Garay, G., \& Lizano, S. 1999, PASP, 111, 1049

Gezari, D. Y., Backman, D. E., \& Werner, M. W. 1998, ApJ, 509, 283

Hayward, T. L., Miles, J. W., Houck, J. R., Gull, G. E., \& Schoenwald, J. 1993, in Infrared Detectors and Instrumentation, ed. A. W. Fowler, Proc. SPIE, 1946, 334

Henning, Th., Feldt, M., Stecklum, B., \& Klein, R. 2001, A\&A, 370, 100

Humphreys, R. M. 1978, ApJS, 38, 909

Keto, E., Proctor, D., Ball, R., Arens, J., \& Jernigan, G. 1992, ApJ, 401, L113

Kurtz, S., Churchwell, E., \& Wood, D. O. S. 1994, ApJS, 91, 659 
Kurtz, S., Cesaroni, R., Churchwell, E., Hofner, P., \& Walmsley, C. M. 2000, in Protostars and Planets IV, ed. V. Mannings, A. P. Boss, \& S. S. Russell (Tucson: University of Arizona Press), 299 Manske, V., \& Henning, Th. 1998, A\&A, 337, 85

Mathis, J. S., Rumpl, W., \& Nordsieck, K. H. 1977, ApJ, 217, 425

Megeath, S. T., \& Tieftrunk, A. R. 1999, ApJ, 526, L113

Osorio, M., Lizano, S., \& D’Alessio, P. 1999, ApJ, 525, 808

Pantin, E., \& Starck, J.-L. 1996, A\&AS, 118, 575

Reid, M. J., Argon, A. L., Masson, C. R., Menten, K. M., \& Moran, J. M. 1995, ApJ, 443, 238

Roelfsema, P. R., Cox, P., Tielens, A. G. G. M., et al. 1996, A\&A, 315, L289

Sandell, G. 2000, A\&A, 358, 242

Tapia, M., Persi, P., \& Roth, M. 1996, A\&A, 316, 102
Thompson, R. I. 1984, ApJ, 283, 165

Turner, J. L., \& Welch, W. J. 1984, ApJ, L81

van der Tak, F. F. S., van Dishoeck, E. F., Evans, N. J., \& Blake, G. A. 2000, ApJ, 537, 283

Wilner, D. J., Welch, W. J., \& Forster, J. R. 1995, ApJ, 449, L73

Wilner, D. J., Reid, M. J., \& Menten, K. M. 1999, ApJ, 513, 775

Wink, J. E., Duvert, G., Guilloteau, S., Walmsley, C. M., \& Wilson, T. L. 1994, A\&A, 281, 505

Wood, D. O. S., \& Churchwell, E. 1989, ApJS, 69, 831

Wyrowski, F., Hofner, P., Schilke, P., et al. 1997, A\&A, 320, L17

Wyrowski, F., Schilke, P., Walmsley, C. M., \& Menten, K. M. 1999, ApJ, 514, L43

Zeng, Q., Hermsen, W., Wilson, T., \& Batrla, W. 1984, A\&A, 140, 169 